\title{
Construction of Mechanic Regulation of Turbine Ventilator using Half-Flap
}

\author{
Josef Sedlak $^{1}$, Jiri Malasek ${ }^{2}$, Martin Ondra ${ }^{2}$, Ales Polzer ${ }^{1}$ \\ ${ }^{1}$ Department of Machining Technology, Institute of Manufacturing Technology, Faculty of Mechanical Engineering, \\ Brno University of Technology, Technická 2896/2, Brno 616 69, Czech Republic. E-mail: sedlak@fme.vutbr.cz, \\ polzer@fme.vutbr.cz \\ ${ }^{2}$ Department of Handling and Building Machines, Institute of Automotive Engineering, Faculty of Mechanical Engineer- \\ ing, Brno University of Technology, Technická 2896/2, Brno 616 69, Czech Republic. E-mail: malasek@fme.vutbr.cz, \\ m.rtin@seznam.cz
}

\begin{abstract}
An article deals with a definition, concept, development, calculation and construction of a prototype solution of a mechanical regulation of a turbine ventilator using a half-flap eliminating an amount of ventilated air. The whole mechanical regulation solution lies in a central shaft. When a rotation nozzle is spun to high revolutions, a flap closes gradually and eliminates air flowing. This prototype of the mechanical regulation may be used for classic concepts of turbine ventilators, where the rotation nozzle stays in one position and only rotates around its own axis.

The article describes individual development stages from the concept up to construction, including a final visualization of the prototype solution of the mechanical regulation of the turbine ventilator using the half-flap.

The mentioned innovative solution of the mechanical regulation is very up-to-date thanks to its simplicity and nonservice operation. It is just the matter of time when one of the producers would be interested in the turbine ventilator solution and it would be introduced into a market space.
\end{abstract}

Keywords: Turbine Ventilator, Mechanical regulation, Half-flap, Drained Air, Flap, Suction Capacity

\section{Acknowledgement}

The research was supported and co-financed from the project called „TA CR GAMA PP1 TG01010054 - VUT SANCE. “

\section{References}

[1] 2015 VYTOZ-EKO spol. s.r.o. Ventilacni turbiny ZLT. All rights reserved. [Online]. [Seen 27th May 2015]. Available at: http://www.vytozeko.cz/ventilacni-turbiny-zlt/.

[2] ${ }^{0} 2015$ H-tech group s.r.o. Ventilacni turbiny a vetraci ventilatory. All rights reserved. [Online]. [Seen $27^{\text {th }}$ May 2015]. Available at: http://www.vetrani.com/index.php/cs/vv-ventilacni-turbiny.

[3] ${ }^{0} 2015$ Haze $\mathrm{s}$ r.o. Lomanco ventilacni turbina. [Online]. [Seen 27 $7^{\text {th }}$ May 2015]. Available at: http://www.haze.cz/stresni-vetraci-systemy/lomanco-ventilacni-turbina/.

[4] ${ }^{2} 2015$ MOBILAND TRADE s.r.o., Edmonds. Neco o ventilaci. [Online]. [Seen 27 $7^{\text {th }}$ May 2015]. Available at: http://www.ventilacniturbiny.cz/neco-o-ventilaci.

[5] ONDRA, M. (2015). Vyvoj a regulace ventilacnich turbin. Brno 2015. Master Thesis. Brno University of Technology, Faculty of Mechanical Engineering, Institute of Automotive Engineering. 84 p., 15 appendices. Advisor doc. Ing. Jiri Malasek, Ph.D.

[6] SEDLAK, J., MALASEK, J., ONDRA, M., POLZER, A. (2016). Development and Production of Prototype Model of Axial Fan. Manufacturing Technology, Vol. 16, No. 2, p. 436-444. ISSN 1213-2489.

[7] CHUA, C. K., LEONG, K. F., LIM, C. S. (2010). Rapid Prototyping: Principles and Applications. $3^{\text {rd }}$ ed. New Jersey: World Scientific, c2010, 512 pp. ISBN 978-981-277-897-0.

[8] SEDLAK, J., SLANY, M., FIALA, Z., JAROS, A. (2015). Production Method of Implant Prototype of Knee-Joint Femoral Component. Manufacturing Technology, Vol. 15, No. 2, p. 195-204. ISSN 1213-2489.

[9] MARCINCIN, J. N., JANAK, M., MARCINCINOVA, L. N. (2012). Increasing of Product Quality Produced by Rapid Prototyping Technology. Manufacturing Technology, June, Vol. 12, No. 12, p. 71-75. ISSN 1213-2489.

[10] SEDLAK, J., PTACKOVA, M., NEJEDLY, J., MADAJ, M., DVORACEK, J., ZOUHAR, J., CHARVAT, O., PISKA, M. (2013). Material Analysis of Titanium Alloy Produced by Direct Metal Laser Sintering. International Journal of Metalcasting, Vol. 7, No. 2, p. 43-50. ISSN 1939-5981.

[11] Edmonds. Neco o ventilaci. [Online]. [Seen $18^{\text {th }}$ April 2015]. Available at: http://www.ventilacniturbiny.cz/necoo-ventilaci. 
[12] Edmonds. In: Hurricane Brochure. [Online]. [Seen 18 ${ }^{\text {th }}$ April 2015]. Available at: http://www.edmonds.com.au/Edmonds/UploadedFiles/40/400d36aa-8717-47d7-adc8-b5cf04f32686.pdf.

[13] Edmonds. In: Technicke specifikace windmaster, superwhirly. [Online]. [Seen 18 ${ }^{\text {th }}$ May 2015]. Available at: http://www.ventilacniturbiny.cz/technicke-dokumenty/superwhirly/superwhirly-tech-specifikace.pdf.

[14] Edmonds. In: Technicke specifikace supavent, turbobeam. [Online]. [Seen 18 ${ }^{\text {th }}$ May 2015]. Available at: http://www.ventilacniturbiny.cz/technicke-dokumenty/turbobeam/turbobeam-tech-specifikace.pdf.

[15] Edmonds. In: Technicke specifikace turbo ventura, sewer vent. [Online]. [Seen 18 $8^{\text {th }}$ May 2015]. Available at: http://www.ventilacniturbiny.cz/technicke-dokumenty/sewervent/sewervent-tech-specifikace.pdf.

[16] SHIGLEY, J. E., MISCHKE, Ch. R., BUDYNAS, R. G. (2010). Konstruovani strojnich soucasti. Brno: VUTIUM, 2010. 1186 p. ISBN 978-80-214-2629-0.

[17] LEINVEBER, J., VAVRA, P. (2011). Strojnicke tabulky. $5^{\text {th }}$ Ed. Uvaly: ALBRA, 2011. 927 p. ISBN 978-807361-081-4.

[18] Pruziny Cermak. [Online]. [Seen 18 th April 2015]. Available at: http://www.pruzinycermak.cz/index_soubory/Page 537.htm.

[19] MITCalc. [Online]. [Seen $18^{\text {th }}$ May 2015]. Available at: http://mitcalc.com/doc/springs/help/cz/springstxt.htm.

\section{Paper number: M2016253}

Copyright (C) 2016. Published by Manufacturing Technology. All rights reserved. 